\title{
Avaliação de Cardápios de Uma Escola de Educação Infantil
}

\author{
Tainara Boito ${ }^{1}$, Valeria Hartmann², Daiana Argenta Kümpel ${ }^{3}$, Graziela De Carli ${ }^{4}$
}

\begin{abstract}
RESUMO
Este estudo teve por objetivo verificar a adequação do cardápio de uma escola municipal de educação infantil do município de Ibiraiaras - RS -, com as preconizações do Programa Nacional de Alimentação Escolar. Realizou-se um estudo transversal em uma escola de educação infantil por meio da avaliação do cardápio oferecido aos pré-escolares de acordo com as recomendações do PNAE e do método de Avaliação Qualitativa das Preparações do Cardápio (AQPC), e também foi verificada a aceitabilidade utilizando a escala hedônica facial. Observou-se que os cardápios oferecidos aos escolares se apresentavam insuficientes na oferta da maioria dos nutrientes abordados pelo PNAE. De acordo com o método AQPC, pode-se perceber a oferta diária de frutas e folhosos aos alunos e uma baixa ocorrência de carne gordurosa e alimentos ricos em enxofre nas refeições. O teste de aceitabilidade foi realizado com 61 escolares, dos quais prevaleceu o sexo feminino (57\%), quando se obteve boa aceitação pelos alunos com relação à alimentação oferecida, com média de $87 \%$ de aceitabilidade. Vale ressaltar que a alimentação ofertada na escola pode exercer influência positiva no rendimento escolar, uma vez que aumenta a capacidade de concentração dos alunos. Apesar de ter uma boa aceitabilidade, os cardápios oferecidos aos escolares devem ser revistos para adequação da oferta de macro e micronutrientes de acordo com as recomendações do PNAE, para que sejam consumidos nutrientes necessários para o crescimento e desenvolvimento adequados das crianças.
\end{abstract}

Palavras-chave: Alimentação infantil. Alimentação escolar. Hábitos alimentares.

\section{MENU EVALUATION OF AN EARLY CHILDHOOD EDUCATION SCHOOL}

\section{ABSTRACT}

The objective of this study was to verify the adjustment of a menu in a municipal early childhood school in the town of Ibiraiaras - RS -, considering the recommendations of the National Food School Program. A transversal study was carried out through the evaluation of a menu offered to the children, according to the PNAE recommendations and the Qualitative Evaluation method of the Menu Preparations (AQPC). Yet, it was verified the acceptability by using the facial hedonic scale. It was observed that the menu offered to those children did not show the majority of nutrientes approached by the PNAE. According to the AQPC method, it was observed the daily servings of fruits and vegetables that are offered to the students and the little quantity of fatty meat and food which are rich in sulfur in their meals. The acceptability test was carried out with 61 children, of which the female prevailed (57\%), in which they seemed to accept well the meals offered to them, showing an average of $87 \%$ acceptability. It is important to emphasize that the food offered in this school may influence positively the students' school performance, since it improves their ability to concentrate. Despite having good acceptability, the menu offered to them must be reviewed for a better adequacy of macro and micronutrientes, according to the PNAE recommendations, so that they offer the necessary nutrients for the children's adequate growth and development.

Keywords: Children's food. School food. Food habits.

Recebido em: 9/4/2018

Aceito em: 25/4/2019

\footnotetext{
Graduação em Nutrição pela Universidade de Passo Fundo. taai_boito@hotmail.com

Graduação em Nutrição pela Universidade Federal de Pelotas (1989). Especialização em Economia e Gestão Empresarial pela Universidade de Passo Fundo (2003). Especialização em Tecnologia de Frutas e Hortaliças (2011). Mestrado em Bioexperimentação pelo Programa de Pós-Graduação em Bioexperimentação da UPF (2014). Professora-adjunta I da UPF e coordenadora do curso de Nutrição. Possui experiência na área de Nutrição, com ênfase em UAN e Técnica Dietética, atuando, principalmente, nos seguintes temas: Alimentação Coletiva, Alimentos, Qualidade, Boas Práticas em Serviços de Alimentação, Higiene e Cardápio. vhartmann@upf.br

${ }^{3}$ Graduação em Nutrição pela Universidade de Cruz Alta (2006). Pós-Graduação em Tecnologia e Controle de Qualidade em Alimentos pela Universidade de Passo Fundo (2008). Mestrado em Envelhecimento Humano pela Universidade de Passo Fundo (2012). Docente do curso de Nutrição da Universidade de Passo Fundo e da Residência Multiprofissional Integrada em Saúde do Idoso e Atenção ao Câncer da Universidade de Passo Fundo (UPF), Hospital São Vicente de Paulo (HSVP) e Prefeitura de Passo Fundo (PMPF). Possui experiência na área de Nutrição e Pesquisa, atuando principalmente nos seguintes temas: Envelhecimento Humano, Geriatria, Oncologia e Saúde Pública. daianakumpel@upf.br

${ }^{4}$ Graduação em Nutrição (2007) e Especialização em Gestão de Pessoas (2009) pela Universidade de Passo Fundo. Especialização em Tecnologia de Frutas e Hortaliças pela Universidade Federal de Pelotas (2011). Especialização em Gastronomia pela Universidade do Vale do Itajaí (2012) e Mestrado em Ciência de Tecnologia de Alimentos pela Universidade de Passo Fundo (2016). Docente da UPF, ministrando disciplinas no curso de Nutrição e no curso Superior de Tecnologia em Estética e Cosmética. Possui experiência na área de Nutrição, com ênfase em Administração de Unidades de Alimentação e Nutrição e Gastronomia. grazieladecarli@upf.br
} 


\section{INTRODUÇÃO}

O Ministério da Educação prevê que as escolas de educação infantil devem proporcionar condições de garantia para o desenvolvimento do potencial de crescimento adequado e a manutenção da saúde integral das crianças (BRASIL, 2012). Entre estes fatores encontra-se a alimentação, que deve atender a todas as exigências do corpo, pois, além de ser fonte de nutrientes, envolve diferentes aspectos, como valores culturais, sociais, afetivos e sensoriais (BASAGLIA; MARQUES; BENATTI, 2015).

A formação de hábitos alimentares saudáveis é um processo que se inicia desde o nascimento, com práticas alimentares introduzidas nos primeiros anos de vida pelos pais (VITOLO, 2008), e que se modificam com a incorporação de novas práticas alimentares durante a pré-escola (PHILIPPI; CRUZ; COLLICI, 2003). A elaboração de cardápios adequados pode ser usada como ferramenta de educação nutricional, pois os alimentos neles presentes podem servir como exemplo para uma alimentação saudável e equilibrada (BOAVENTURA; OLIVEIRA, 2013).

Em virtude da importância da alimentação oferecida aos alunos, o governo brasileiro, em 1955, criou o Programa Nacional de Alimentação Escolar (PNAE), fundamentado no direito dos escolares de ter acesso a uma alimentação segura, saudável e adequada. 0 programa orienta que o cardápio seja elaborado por nutricionista responsável e forneça, no mínimo, 20\% do valor energético total, quando servida apenas uma refeição por dia para alunos matriculados na educação básica em período parcial, sendo responsável pelo suprimento das necessidades dos escolares em macronutrientes e micronutrientes (BRASIL, 2009). Nesse contexto, a elaboração dos cardápios constitui a principal ação ligada, efetivamente, à alimentação escolar (SOUSA et al., 2015).

Estudo de Inoue et al. (2015) evidenciou inadequações nos cardápios oferecidos em relação a alguns micronutrientes e um elevado consumo energético na alimentação nos centros municipais de educação infantil de Colombo - PR, e ratifica a importância de adequar o consumo alimentar e promover hábitos de alimentação saudável com as crianças de creches públicas.

Outro fator importante é a aceitabilidade dos alimentos pelos escolares, pois é um aspecto determinante da qualidade do serviço prestado pelas escolas em relação ao fornecimento da alimentação escolar (BRASIL, 2010). Para Basaglia, Marques e Benatti (2015), as preparações podem ser consideradas de boa aceitabilidade quando preenchem as expectativas da maior parte dos alunos com relação às características sensoriais, como aparência, cor, aroma, textura e sabor. Estudos indicam que a aceitabilidade dos escolares referente à alimentação ofertada é boa, porém, no que diz respeito à recomendação do PNAE, observa-se que estas se encontram insuficientes (BASAGLIA; MARQUES; BENATTI, 2015; NUNES, 2014).

A qualidade das preparações pode ser avaliada pelo método de avaliação Qualitativa das Preparações do Cardápio (AQPC), que consiste em uma análise do cardápio que engloba a qualidade nutricional e sensorial das preparações. Menegazzo et al. (2011) observaram que a alimentação oferecida aos pré-escolares em centros de educação infantil, no almoço e no jantar, apresenta baixa oferta de carnes gordurosas e frituras, sem excesso de alimentos sulfurados tampouco monotonia de cores. Há, porém, alta oferta de carboidratos simples e ácidos graxos trans, pouca oferta de frutas, legumes e verduras e monotonia de cores nos lanches (MENEGAZZO et al., 2011).

Com o intuito de colaborar para a alimentação saudável na infância, o presente estudo teve como objetivo verificar a adequação do cardápio de uma escola municipal de educação infantil do município de Ibiraiaras - RS - de acordo com as preconizações do PNAE.

\section{MÉTODO}

Trata-se de um estudo transversal em uma escola de educação infantil do município de Ibiraiaras - RS. Avaliou-se o cardápio oferecido aos pré-escolares de acordo com as recomendações do PNAE e por meio do método de Avaliação Qualitativa das Preparações do Cardápio (AQPC), bem como a aceitabilidade, no mês de julho de 2016. O estudo foi aprovado pelo Comitê de Ética em Pesquisa da UPF - paracer no 1.553.685.

Foi acompanhado o preparo das refeições pelas merendeiras no período de cinco dias, durante o preparo dos lanches dos turnos da manhã e tarde, posto que os alimentos utilizados para o preparo foram previamente pesados em balança digital, com capacidade mínima de pesagem de $2 \mathrm{~g}$ e máxima de $15 \mathrm{~kg}$. Após foram calculados os per capitas, dividindo-se o peso de cada alimento pelo número de lanches oferecidos em cada turno, e também foi realizada uma avaliação do cardápio pelo cálculo da composição nutricional, utilizando a Tabela de Composição Química de Alimentos (Taco).

Os cardápios calculados foram avaliados de acordo com as recomendações do PNAE, utilizando os parâmetros para o atendimento de, no mínimo, 
$20 \%$ das necessidades nutricionais diárias de macronutrientes e micronutrientes dos alunos beneficiários, considerando que os mesmos realizam apenas uma refeição na escola, além de comparar com as recomendações de alimentos e suas quantidades, que devem estar presentes no cardápio escolar. Neste, foi avaliada a oferta de, pelo menos, três porções de frutas e hortaliças por semana, além do limite máximo de $10 \%$ do valor energético proveninte de açúcar simples, $15 \%$ a $30 \%$ proveniente de gorduras totais, sendo $10 \%$ de gordura saturada, $1 \%$ de gordura trans e uma grama de sal por dia (BRASIL, 2009).

Para a execução do teste de aceitabilidade foi autorizada a participação das crianças pelos pais ou responsáveis mediante o Termo de Consentimento Livre e Esclarecido e, para verificar o índice de aceitabilidade da alimentação oferecida aos escolares, foi utilizada a escala hedônica facial, a qual indica o grau de satisfação em relação à preparação servida na escola. Para o cálculo do índice de aceitabilidade foi considerado o somatório das porcentagens de respostas positivas sobre a preparação, contemplando os elementos gostei e adorei. Para serem considerados de boa aceitabilidade, o teste de aceitabilidade pela escala hedônica facial deve ter seu índice de 85\% (BRASIL, 2009).

O método de Avaliação Qualitativa das Preparações do Cardápio (AQPC), proposto por Proença et al. (2005), foi utilizado para avaliar o cardápio em relação à monotonia de cores, presença de folhosos, carnes gordas, frituras, gordura associada a doces, alimentos ricos em enxofre, frutas, legumes e verduras nas preparações servidas aos escolares. A refeição foi considerada rica em enxofre, quando havia a presença de um ou mais alimentos sulfurados, com exceção do feijão, que é recomendado consumo diário (BRASIL, 2006). Para esta avaliação foram registrados os cardápios de todos os dias do mês de julho de 2016, digitados no software Excel 2007, e avaliados conforme a ocorrência dos itens estabelecidos no cardápio.

As condições socioecônomicas dos participantes foram avaliadas com base no Critério de Classificação Econômica Brasil da Associação Brasileira de Empresas de Pesquisa (Abep), a partir de um questionário enviado aos pais juntamente com o TCLE (BRASIL, 2015).

\section{RESULTADOS E DISCUSSÃO}

Na Tabela 1 encontra-se a média dos resultados da avaliação da composição nutricional dos cardápios servidos na escola, que foram comparados com as recomendações do PNAE (BRASIL, 2009) para o atendimento de, no mínimo, $20 \%$ das necessidades nutricionais de pré-escolares. Nos resultados verificou-se que os lanches oferecidos aos escolares matriculados no turno da manhã e tarde apresentaram-se deficientes em nutrientes essenciais para o crescimento e desenvolvimento saudável da criança, tais como proteínas, lipídios, fibras, cálcio, magnésio, zinco e vitamina $A$, assim como o teor calórico das preparações, apresentando quantidades adequadas para carboidratos, ferro e gordura saturada, posto que os valores recomendados para gordura saturada devem estar abaixo do limite máximo (10\%). A oferta de vitamina C foi adequada para o turno da manhã, no entanto para o turno da tarde foi insuficiente.

Tabela 1 - Recomendação do PNAE e resultado médio da composição nutricional dos cardápios dos lanches da manhã (Lanche I) e da tarde (Lanche II) da escola avaliada

\begin{tabular}{|l|c|c|c|c|c|}
\hline \multicolumn{1}{|c|}{ Nutrientes } & PNAE & $\begin{array}{c}\text { Lanche } \\
\text { I }\end{array}$ & $\begin{array}{c}\text { Ade- } \\
\text { quação } \\
\text { (\%) }\end{array}$ & $\begin{array}{c}\text { Lanche } \\
\text { II }\end{array}$ & $\begin{array}{c}\text { Ade- } \\
\text { quação } \\
\text { (\%) }\end{array}$ \\
\hline Calorias & 270,0 & 244,0 & 90,2 & 207,0 & 76,8 \\
\hline Proteína (g) & 8,4 & 4,6 & 55,0 & 4,5 & 53,2 \\
\hline Lipídeo(g) & 6,8 & 3,2 & 47,2 & 1,8 & 25,9 \\
\hline Carboidrato (g) & 43,9 & 49,1 & 111,8 & 43,4 & 98,9 \\
\hline Fibra (g) & 5,0 & 1,2 & 24,6 & 1,1 & 23 \\
\hline Cálcio (mg) & 160,0 & 35,4 & 22,1 & 15,5 & 9,7 \\
\hline Magnésio (mg) & 26,0 & 14,3 & 54,9 & 12,4 & 47,6 \\
\hline Ferro (mg) & 2,0 & 2,2 & 108,2 & 2,0 & 100,0 \\
\hline Sódio (mg) & 400,0 & 283,4 & 70,9 & 248,5 & 62,1 \\
\hline Zinco (mg) & 1,0 & 0,3 & 26,8 & 0,4 & 44,3 \\
\hline Retinol (mg) & 80,0 & 4,2 & 5,2 & 60,4 & 75,5 \\
\hline Vitamina C (mg) & 5,0 & 6,0 & 119,3 & 1,6 & 32,1 \\
\hline G. Saturada (\%) & 10 & 1,1 & 35,0 & 0,5 & 27,8 \\
\hline
\end{tabular}

Uma pesquisa realizada por Pegolo e Silva (2010) avaliou o consumo energético e nutricional e a adesão ao PNAE por escolares de um município paulista, encontrando resultados semelhantes com o do presente estudo, verificando que cerca de metade dos escolares apresentaram consumo insuficiente de energia bem como de vitaminas e minerais. Bezerra e Coradi (2016) encontraram inadequações energéticas, de cálcio, sódio e Vitamina A na alimentação oferecida aos escolares em turnos. Silva e Gregório (2012), entretanto, avaliaram a composição nutricional dos cardápios de todas as escolas da rede municipal de ensino do município de Taquaraçu de Minas - MG quanto ao cumprimento das exigências nutricionais do PNAE para crianças e adolescentes, e perceberam que os cardápios oferecidos na alimentação escolar do município atingem, em sua maioria, as recomendações do PNAE para crianças, mas são parcialmente insuficientes para os adolescentes quanto ao teor 
calórico, glicídico, proteico, de cálcio, magnésio e de zinco, além de ultrapassar a recomendação para vitamina $\mathrm{A}$, vitamina $\mathrm{C}$ e lipídeos.

Inadequações no consumo de nutrientes podem comprometer o estado nutricional e levar ao desenvolvimento de carências ou excessos nutricionais (CAVALCANTE et al., 2006). Pode-se destacar o cálcio, que, dentre todos os micronutrientes avaliados em ambos os estudos, foi o que esteve em maior desacordo com as necessidades infantis e com as recomendações do PNAE. Considerando que o aporte deste mineral esteja inadequado, respostas negativas podem incidir no desenvolvimento de crianças, principalmente durante o crescimento. Assim, é importante garantir ingestão mínima de cálcio para o completo crescimento e maturação dos ossos (ABRANCHES et al., 2009).

$\mathrm{Na}$ avaliação da quantidade de alimentos oferecidos aos pré-escolares, foi observado que a oferta de frutas e hortaliças era menor que meia porção diária, considerada insuficiente. Levando isso em conta, o baixo teor de fibras encontrado na Tabela 1 está relacionado à baixa oferta de frutas, verduras e legumes às crianças, o que é preocupante e exige ações para o aumento desse consumo, uma vez que esses alimentos são essenciais para o desenvolvimento infantil por serem fontes de vitaminas, minerais e fibras alimentares (BRASIL, 2006). Bezerra e Coradi (2016) evidenciam, em seu estudo, que a menor quantidade de alimentos ofertada pode estar relacionada com as inadequações de nutrientes essenciais na alimentação.

A quantidade de gordura total, gordura saturada e de sódio também ficou abaixo da recomendação, e não foi encontrada a presença de gordura trans na alimentação dos escolares, sendo estes fatores positivos ao cardápio. A quantidade de açúcar simples adicionado, no entanto, ficou próximo ao máximo recomendado (9\%). Dessa forma, os resultados evidenciam inadequações às recomendações nutricionais do PNAE, com deficiências de macronutrientes e micronutrientes importantes na participação de mecanismos biológicos, como crescimento, desenvolvimento cognitivo e imunológico dos escolares. Essa inadequação pode ser consequência de um planejamento incorreto, tanto do ponto de vista dos ingredientes escolhidos quanto do dimensionamento das quantidades per capita (ISSA et al., 2014).

O teste de aceitabilidade foi realizado com média de 49 pré-escolares, dos quais prevaleceu o sexo feminino (57\%). A maioria dos escolares (43\%) foi classificada no nível socioecônomico $C$, seguidos por B (38\%), D (16\%) e A (3\%). No resultado da avaliação obteve-se boa aceitação pelos alunos com relação à alimentação oferecida, com média de $87 \%$ de aceitabilidade, estando acima da recomendação do PNAE, conforme mostra a Tabela 2.

Um estudo realizado na cidade de Amparo - SP - com crianças e adolescentes, obteve uma aceitabilidade menor que o encontrado na escola de educação infantil de Ibiraiaras - RS -, posto que a merenda escolar foi considerada boa por parte dos alunos do $2 \circ$ ao 50 anos do Ensino Fundamental $(83,92 \%)$, enquanto a aceitabilidade manifestada pelos escolares do 5으 ao 3응 anos do Ensino Médio foi menor (74,14\%) (BASAGLIA; MARQUES; BENATTI, 2015). Este resultado é semeIhante às pesquisas realizadas em escolas com crianças e adolescentes, que mostram que a aceitabilidade difere de uma faixa etária para outra, obtendo sempre uma maior aceitabilidade com as crianças (BASAGLIA; MARQUES; BENATTI, 2015; GARCIA et al., 2016).

Tabela 2 - Resultado do teste de aceitabilidade realizado por meio da escala hedônica facial

\begin{tabular}{|c|c|c|c|c|c|c|c|}
\hline \multirow{2}{*}{ Data } & $\begin{array}{c}\text { Total de } \\
\text { escolares }\end{array}$ & \multicolumn{2}{|c|}{$\begin{array}{c}\text { Adorei + } \\
\text { gostei }\end{array}$} & \multicolumn{2}{|c|}{ Indiferente } & \multicolumn{2}{|c|}{$\begin{array}{c}\text { Não gostei } \\
\text { + Odiei }\end{array}$} \\
\cline { 2 - 9 } & & $\mathbf{( N )}$ & $\mathbf{\%}$ & $\mathbf{( N )}$ & $\mathbf{\%}$ & $\mathbf{( N )}$ & $\%$ \\
\hline 1 & 56 & 50 & 89,3 & 1 & 1,8 & 4 & 7,1 \\
2 & 54 & 48 & 88,9 & 2 & 3,7 & 4 & 7,4 \\
\hline 3 & 50 & 42 & 84,0 & 3 & 6,0 & 5 & 10,0 \\
\hline 4 & 34 & 30 & 88,2 & 3 & 8,8 & 1 & 2,9 \\
\hline 5 & 53 & 46 & 86,8 & 3 & 5,7 & 4 & 7,5 \\
\hline MÉDIA & $\mathbf{4 9}$ & $\mathbf{4 3}$ & $\mathbf{8 7}$ & $\mathbf{2}$ & $\mathbf{5}$ & $\mathbf{4}$ & $\mathbf{7}$ \\
\hline
\end{tabular}

Além disso, um estudo recente mostrou a importância da família no comportamento das crianças, ao constatar que os alunos com maior chance de aceitar a alimentação escolar são aqueles cujos pais acreditam na qualidade da refeição servida pela escola (OHRI, 2014).

Apesar de ter uma boa aceitabilidade, a avaliação do cardápio mostrou deficiências importantes de macronutrientes e micronutrientes. Diante disso, faz-se necessário priorizar e avaliar os alimentos que estão sendo oferecidos nas escolas infantis, de modo a verificar se estes se encontram adequados para o desenvolvimento físico e mental das crianças que os consomem, levando em consideração que os hábitos alimentares nos primeiros anos irão repercutir de diferentes formas ao longo de toda a vida dos indivíduos (SILVA et al., 2016).

Dessa forma, as preferências alimentares são aprendidas a partir de novas experiências repetidas do consumo de determinado alimento (VITOLO, 2008), por isso é importante que a criança seja exposta a diferentes tipos de alimentos desde a introdução 
alimentar, o que contribui na aceitação de novos sabores, uma vez que as crianças possuem tendência a rejeitá-los (GIULIANI; VICTORA, 2000).

Considerando que a alimentação escolar representa uma relação direta com a qualidade de vida do aluno, e influencia de forma determinante no seu rendimento escolar por propiciar condições que favorecem a capacidade de concentração e, consequentemente, a aprendizagem (NASCIMENTO et al., 2016), as escolas devem assumir uma posição de incentivo à promoção de comportamentos alimentares que auxiliem a formação de hábitos saudáveis, assegurando a oferta de alimentos e refeições nutricionalmente equilibrados aos escolares e de boa aceitabilidade. Vale ressaltar que a alimentação oferecida na escola pode exercer influência positiva no rendimento escolar, uma vez que aumenta a capacidade de concentração dos alunos (BRASIL, 2012).

O método AQPC foi aplicado utilizando o cardápio oferecido aos pré-escolares nos lanches e aos professores, funcionários e escolares de turno integral no horário de almoço. De acordo com este, pode-se perceber a oferta diária de frutas e folhosos aos alunos, e observou-se uma baixa ocorrência de carne gordurosa. Quanto à oferta de doces, ocorreu em um terço dos dias avaliados, e alimentos ricos em enxofre esteviveram presentes em $42 \%$ dos dias avaliados. Não houve oferta de frituras, alimentos de cores iguais e doces associados a fritura.

Os resultados encontrados na presente pesquisa (Tabela 3) são similares aos verificados no estudo de Boaventura e Oliveira (2013), que avaliou as preparações dos cardápios de escolas municipais da Grande São Paulo pelo método AQPC, aplicado nas cidades de Barueri, Guarulhos, São Bernardo do Campo e São Paulo (SP). Foi encontrada oferta adequada de hortaliças e frutas, entretanto a presença de doces e alimentos ricos em enxofre é constante e, por isso, os autores argumentam que o método é um bom instru- mento para a avaliação da qualidade do cardápio e sugerem que sejam revistas a oferta de alimentos sulfurados e carboidratos simples.

\section{CONCLUSÕES}

Sabe-se que a alimentação na infância assume caráter decisivo na formação de hábitos alimentares que podem perdurar na vida adulta. Dessa forma, deve haver incentivo para a formação de hábitos alimentares saudáveis, principalmente nessa faixa etária.

Neste estudo, foi identificada uma boa aceitabilidade pelos pré-escolares em relação ao cardápio oferecido na escola. Também foi observado que a alimentação oferecida se encontrava inadequada em relação às recomendações nutricionais, com deficiência na maioria dos nutrientes analisados. Quanto às quantidades recomendadas pelo programa, o cardápio dos lanches da escola apresentou-se parcialmente adequado. De acordo com o método AQPC, o planejamento dos cardápios do almoço da escola municipal, em geral, mostrou-se adequado, mas deve-se atentar para a prevalência de doces e carnes gordurosas, de modo a melhor atender às necessidades das crianças.

Diante do exposto, os cardápios oferecidos aos pré-escolares devem ser revistos para adequação da oferta de nutrientes de acordo com as recomendações do PNAE, para que sejam supridos os nutrientes necessários para o crescimento e desenvolvimento adequados das crianças.

\section{COLABORADORES}

- Tainara Boito e Valeria Hartmann desenvolveram o projeto, análise dos dados e resultados.

- Tainara Boito, Valeria Hartmann, Graziela De Carli e Daiana Argenta Kümpel escreveram e revisaram o artigo.

Tabela 3 - Resultado da Avaliação Qualitativa das Preparações do Cardápio (AQPC) de um mês da escola avaliada

\begin{tabular}{|c|c|c|c|c|c|c|c|c|c|}
\hline Semana & $\begin{array}{c}\text { Dias de } \\
\text { cardápio }\end{array}$ & Frituras & Frutas & Folhosos & $\begin{array}{c}\text { Cores } \\
\text { iguais }\end{array}$ & $\begin{array}{c}\text { Ricos em } \\
\text { enxofre }\end{array}$ & $\begin{array}{c}\text { Carne } \\
\text { gordurosa }\end{array}$ & Doce & $\begin{array}{c}\text { Doce e } \\
\text { fritura }\end{array}$ \\
\hline 1 & 5 & 0 & 5 & 5 & 0 & 3 & 1 & 1 & 0 \\
\hline 2 & 5 & 0 & 5 & 5 & 0 & 1 & 2 & 2 & 0 \\
\hline 3 & 4 & 0 & 4 & 4 & 0 & 2 & 1 & 2 & 0 \\
\hline 4 & 5 & 0 & 5 & 5 & 0 & 2 & 0 & 2 & 0 \\
\hline Total de dias & 19 & 0 & 19 & 19 & 0 & 8 & 4 & 7 & 0 \\
\hline \% de ocorrência & 100 & 0 & 100 & 100 & 0 & 42 & 21 & 36 & 0 \\
\hline
\end{tabular}




\section{REFERÊNCIAS}

ABRANCHES, M. V. et al. Avaliação da adequação alimentar de creches pública e privada no contexto do programa nacional de alimentação escolar. Revista Nutrire, São Paulo, v. 34, n. 2, p. 43-57, ago. 2009.

BASAGLIA, P.; MARQUES, A. S.; BENATTI, L. Aceitação da merenda escolar entre alunos da rede estadual de ensino da cidade de Amparo - SP. Revista Saúde em Foco, Rio de Janeiro, v. 7, p. 126-138, 2015.

BRASIL. Associação Brasileira de Empresas de Pesquisa (Abep). Critério de classificação econômica. 2015.

BRASIL. Ministério da educação; Cecane. Manual para aplicação dos testes de aceitabilidade no Programa Nacional de Alimentação Escolar - PNAE. São Paulo: Unifesp, 2010.

BRASIL. Ministério da Educação. Fundo Nacional de Desenvolvimento da Educação. Resolução/CD/FNDE n. 38, de 16 de julho de 2009. Dispõe sobre o atendimento da alimentação escolar aos alunos da educação básica no Programa Nacional de Alimentação Escolar - PNAE. Brasília, DF: MEC, 2009.

BRASIL. Ministério da Educação. Manual de orientação para a alimentação escolar na educação infantil, Ensino Fundamental, Ensino Médio e na Educação de Jovens e Adultos. Organizadores Francisco de Assis Guedes de Vasconcelos et al. 2. ed. Brasília: PNAE; Cecane-SC, 2012.

BRASIL. Ministério da Saúde. Secretaria de Atenção à Saúde. Guia alimentar para a população brasileira: promovendo a alimentação saudável. Brasília: MS, 2006.

BEZERRA, A. S.; CORADI, F. B. Análise da alimentação oferecida para crianças em uma creche municipal do Rio Grande do Sul. Revista Contexto \& Saúde, ljuí: Ed. Unijuí, v. 16, n. 31, 2016.

BOAVENTURA, P. S.; OLIVEIRA, A. C. et al. Avaliação qualitativa de cardápios oferecidos em escolas de educação infantil da grande São Paulo. Demetra: Alimentação, Nutrição \& Saúde, São Paulo, v. 8, n. 3, 2013.

CAVALCANTE, A. A. M. et al. Consumo alimentar e estado nutricional de crianças atendidas em serviços públicos de saúde do município de Viçosa, Minas Gerais. Revista de Nutrição, Campinas, v. 19, n. 3, p. 321-330, 2006.

GARCIA, L. S. et al. Aceitabilidade da alimentação escolar por crianças e adolescentes de um município rural do nordeste brasileiro. Revista Científica da Escola da Saúde, Natal, v. 5, n. 1, p. 85-94, 2016.

GIULIANI, E. R.; VICTORA, C. G. Artigo de revisão: alimentação complementar. Jornal de Pediatria, Porto Alegre, v. 76, n. 3, p. 253-262, 2000.
INOUE, D. Y. et al. Consumo alimentar de crianças de 12 a 30 meses que frequentam centros municipais de educação infantil no município de Colombo, Sul do Brasil. Revista de Nutrição, Campinas, v. 28, n. 5, p. 523-532, 2015.

ISSA, R. C. et al. Alimentação escolar: planejamento, produção, distribuição e adequação. Revista Panamericana de Salud Publica, São Paulo, v. 35, n. 2, p. 96-103, 2014.

MENEGAZZO, M. et al. Avaliação qualitativa das preparações do cardápio dos centros de educação infantil. Revista de Nutrição, Campinas, v. 24, n. 4, mar./abr. 2011.

NASCIMENTO, M. C. et al. Avaliação da adesão e aceitabilidade dos cardápios do Programa de Alimentação Escolar em escolas municipais de Itapetinga - BA: indicadores de desperdício de alimentos. Revista Eletrônica em Gestão, Educação e Tecnologia Ambiental, Santa Maria, v. 20, n. 1. p. 73-85, 2016.

NUNES, B. G. Q. Observação da aceitação da merenda escolar em uma escola municipal de Uberlândia-MG. Revista E-RAC, Minas Gerais, v. 4, n. 1, p. 1-13, 2014.

$\mathrm{OHRI}, \mathrm{V}$. P. Parental perception of the nutritional quality of school meals and its association with students' school lunch participation. Appetite, n. 74, p. 44-47, 2014.

PEGOLO, G. E.; SILVA, M. V. Consumo de energia e nutrientes e a adesão ao Programa Nacional de Alimentação Escolar (PNAE) por escolares de um município paulista. Revista E-RAC, Minas Gerais, v. 17, n. 2, p. 50-62, 2010.

PHILIPPI, S. T.; CRUZ, A. T. R.; COLLICCI, A. C. A. Pirâmide alimentar para crianças de 2 a 3 anos. Revista de Nutrição, Campinas, v. 16, n. 1, jan./mar. 2003.

PROENÇA, R. P. C. et al. Qualidade nutricional e sensorial na produção de refeições. Florianópolis: Editora da UFSC, 2005.

SILVA, G. A. P. et al. Infant feeding: beyond the nutritional aspects. Jornal de Pediatria, Rio de Janeiro, 3 ed., v. 1, p. 2-7, 2016.

SILVA, M. M. D. C.; GREGÓRIO, E. L. Avaliação da composição nutricional dos cardápios da alimentação escolar das escolas da rede municipal de Taquaraçu de Minas - MG. Revista HU, Juiz de Fora, v. 37, n. 3, p. 387-394. jul./set. 2012.

SOUSA, A. A. et al. Cardápios e sustentabilidade: ensaio sobre as diretrizes do Programa Nacional de Alimentação Escolar. Revista de Nutrição, Campinas, v. 28, n. 2, p. 217-229, 2015.

VITOLO, M. R. Parte IV Infância. In: VITOLO, M. R. Nutrição: da gestação ao envelhecimento. Rio de Janeiro, RJ: Editora Rúbio, 2008. p. 167-264. 